\title{
COMMENT
}

\section{Clinical translation of silica nanoparticles}

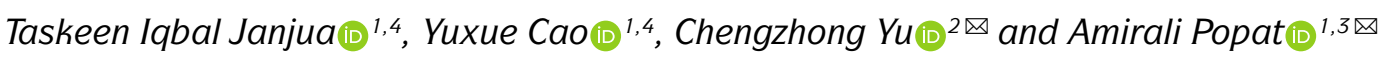

Silica nanoparticles have entered clinical trials for a variety of biomedical applications, including oral drug delivery, diagnostics, plasmonic resonance and photothermal ablation therapy. Preliminary results indicate the safety, efficacy and viability of silica nanoparticles under these clinical scenarios.

Nanoparticle-based therapies have found their way into various clinical applications, with more than 30 nanoformulations approved and over 100 in clinical trials ${ }^{1}$. Furthermore, the expeditious development of two lipid nanoparticle-based COVID-19 mRNA vaccines (BNT162b2 and mRNA-1273) has demonstrated the potential clinical impact of nanoparticle-based therapeutics and initiated a new wave of preclinical and clinical research activities for nanoparticle-based systems. Other examples of approved nanoparticle-based formulations in the clinic include anti-cancer medications (Doxil, Abraxane, Myocet and MEPACT), contrast agents (Resovist), anaesthetics (Diprivan), antifungal drugs (AmBisome), RNAi drugs (ONPATTRO) and other vaccines (Epaxal and Inflexal V).

In addition to lipid nanoparticles, the number of approved inorganic nanoparticles has also substantially increased, making them one of the major classes of clinically used nanoparticles ${ }^{1}$. In particular, silica nanoparticles are being explored as multifunctional delivery carriers in a variety of biomedical applications, including diagnosis, sensing and drug delivery ${ }^{2}$. Mesoporous silica nanoparticles with pore sizes of $2-50 \mathrm{~nm}$ have beneficial properties, such as a large surface area $\left(>1,000 \mathrm{~m}^{2} \mathrm{~g}^{-1}\right)$, and tunable shapes and sizes, making them an ideal platform for diagnosis and as vehicles for drug or gene delivery $^{2}$. Compared with organic nanoparticles, silica nanoparticles have a higher loading capacity, particularly for biologics, good mechanical stability, and they can be tuned to control drug release in response to internal (for example, $\mathrm{pH}$, enzyme and bacteria) and/or external (for example, $\mathrm{pH}$, light, heat and magnetic field) stimuli ${ }^{2}$. As a material, colloidal silica has been used in tablet manufacturing as a glidant for decades and is generally recognized as safe by the US Food and Drug Administration (FDA). In addition, the commonly used food additive E551 is composed of $100 \mathrm{~nm}$ silica nanoparticles. Consequently, various silica nanoparticle-based formulations are now in phase I and phase II clinical trials.

\section{Silica nanoparticles in clinical trials}

The safety profile of silica nanoparticles in humans is evident from 11 clinical trials and 2 clinical studies (FIG. 1). Orally delivered silica nanoparticles have shown good tolerability with no serious adverse effects, while improving the pharmacokinetic profile of hydrophobic drugs $^{3,4}$. In one of the first human studies, a silica-lipid hybrid formulation was used to orally deliver ibuprofen to 16 healthy adults, demonstrating an increase in bioavailability by 1.95 times $^{3}$. In a clinical trial that included 12 adults, lipoceramic hybrid silica nanoparticles were used to improve simvastatin pharmacokinetics, showing a 3.5-fold increase in bioavailability compared with the commercially available simvastatin formulation Sandoz (ACTRN12618001929291) ${ }^{5}$. In another clinical study, in which 12 healthy adults participated, oral delivery of mesoporous silica nanoparticles improved the bioavailability of fenofibrate by $54 \%$, compared with the commercially available fenofibrate formulation Lipanthyl ${ }^{4}$.

In addition to drug delivery, silica nanoparticles have been applied for plasmonic resonance therapy to treat cardiovascular diseases, to detect difficult-to-treat tumours and to thermally ablate tumours ${ }^{6,7}$. Compared with organic nanoparticles (polymeric and lipidic), silica nanoparticles can be easily modified with iron oxide and gold, which can be exploited for localized plasmonic and thermal ablation therapy ${ }^{6,7}$. Results from a completed phase I clinical trial showed that plasmonic resonance therapy using $\mathrm{Fe}_{3} \mathrm{O}_{4}$ ferromagnetic core-shell silicagold nanoparticles (diameter $90-150 \mathrm{~nm}$ ) significantly reduced coronary atherosclerosis (NCT01270139). Moreover, compared with conventional stent implantation, patients treated with hybrid silica nanoparticles had reduced risk of atherosclerosis and cardiovascular disease-related death, with an acceptable level of safety ${ }^{6,7}$.

Further studies were conducted using silica nanoparticles with a gold shell (Aurolase and Auroshell) for the initiation of photothermal ablation of malignant cancer located in the head, neck (NCT00848042) and prostate (NCT04240639, NCT02680535 and NCT04656678) ${ }^{8}$. 


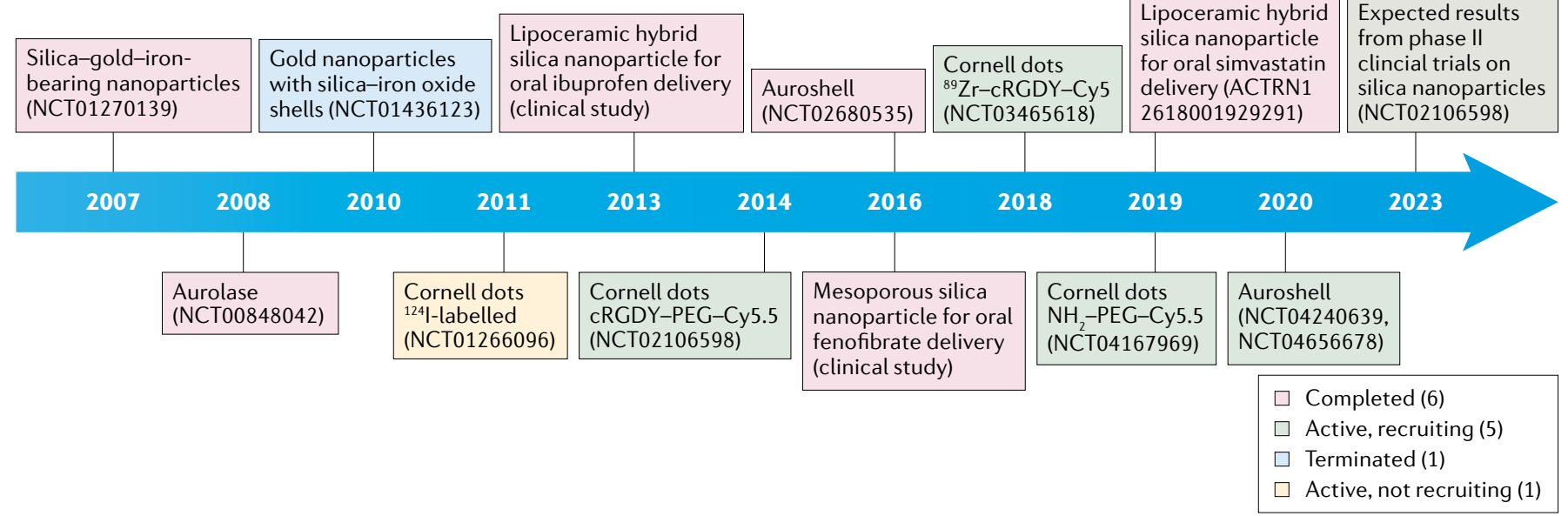

Fig. 1 | Clinical trials of silica nanoparticles. The timeline shows different types of silica nanoparticle in clinical trials. cRGDY, cyclic arginyl-glycyl-aspartic acid-tyrosine; PEG, polyethylene glycol.

These gold shell-silica nanoparticles were designed to convert near-infrared light signals into heat for tumour ablation ${ }^{8}$. In these clinical trials, a $4.8 \mathrm{mg} \mathrm{ml}^{-1}$ solution of gold shell-silica nanoparticles was intravenously injected at a dose of $7.5 \mathrm{ml} \mathrm{kg}^{-1}$; the nanoparticles preferentially accumulated in the tumour via the enhanced permeation and retention effect ${ }^{8}$. Although the results from some clinical trials using these particles to treat prostate cancer are still pending (NCT04240639, NCT02680535 and NCT04656678), a pilot clinical study with 16 individuals with prostate cancer demonstrated successful photothermal ablation using gold shell-silica nanoparticles ${ }^{8}$. The main advantage of gold shellsilica nanoparticles is that they accumulate at the tumour site, allowing accurate and predictable ablation therapy for prostate cancer, with less side effects than conventional focal ablation ${ }^{8}$.

Cornell dots are ultrasmall silica nanoparticles with a diameter of $6-10 \mathrm{~nm}$. Cornell dots can be applied as tracers for tumours, such as melanoma or malignant brain tumours (NCT03465618, NCT01266096 and NCT02106598). The size of Cornell dots is below the $10 \mathrm{~nm}$ renal clearance threshold, and they are thus cleared by the kidneys, addressing concerns of silica nanoparticle accumulation in the body. Cornell dots can be functionalized with tumour-homing arginyl-glycyl-aspartic acid-tyrosine (RGDY) peptide and fluorescent dye Cy5.5 to serve as an imaging agent $^{9,10}$. The first-in-human clinical trial of Cornell dots labelled with ${ }^{124} \mathrm{I}$ demonstrated their application as a hybrid system for positron emission tomography imaging and fluorescent-based detection for the diagnosis and staging of tumours, such as melanoma and malignant brain cancer (NCT01266096) ${ }^{10}$. The preliminary results of this trial demonstrate that Cornell dots are stable, preferentially taken up by the tumour, well tolerated, without any significant side effects, and renally cleared, with an 8.7-hour half-life in plasma ${ }^{10}$. The same Cornell dots were also tested for their ability to detect and localize sentinel lymph nodes in patients with head or neck melanoma to allow visualization during biopsy (NCT02106598) ${ }^{9}$. Clinically used radio-guided sentinel lymph node imaging suffers from low sensitivity, which makes visualization of lymph nodes difficult and therefore surgical biopsy risky ${ }^{9}$. Encapsulating Cy5.5 in silica nanoparticles has been shown to further increase their fluorescent intensity and photostability ${ }^{9}$. Based on these clinical trials, silica nanoparticle-based imaging holds great promise for detecting, staging and biopsy of tumours with high accuracy and low procedural risk.

\section{Towards clinical translation}

Despite demonstrated safety and efficacy from clinical trials (FIG. 1), the rate of clinical translation of silica nanoparticles remains slow. For example, although gold shell-silica nanoparticles were developed in the 1990s, it took about two decades for this technology to advance into clinical trials. Organic nanoparticles, such as liposomes and lipid nanoparticles, have been successfully translated into clinical applications. However, compared with organic nanoparticles, silica nanoparticles have several advantages, which warrant further clinical studies; for example, by modulating their structural and physiochemical properties, such as size, charge, surface functionality and shape, silica nanoparticles can deliver drugs across biological barriers. Moreover, they can easily be hybridized with other inorganic particles, such as gold nanoparticles. Silica nanoparticles also possess self-adjuvant properties, which is desirable for the development of next-generation nanovaccines ${ }^{2}$. In addition, silica nanoparticles are stable in harsh biological settings, for example, in the acidic environment of the stomach, in which liposomes usually degrade, limiting their applicability in oral delivery ${ }^{2}$. Consequently, silica nanoparticles with good safety, efficacy and viability may be applicable in more diverse clinical scenarios than their organic counterparts.

Based on their performance in clinical trials, silica nanoparticles are emerging as a promising diagnostic and delivery platform and could play a key role in the development of next-generation theranostics, nanovaccines and formulations to orally deliver peptides and proteins. However, key hurdles that remain to be 
overcome for silica nanoparticles' clinical translation include establishing safety from chronic exposure, establishing long-term toxicological profiles from different routes of administration, investigating reliable scale-up methods and synthesizing reproducible silica nanoparticles with minimal batch-to-batch variation. Moreover, only solid silica nanoparticles without pores or with small pores have been clinically tested thus far. These nanoparticles do not have high cargo-loading capacity, especially for nucleic-acid-based drugs. Alternatively, dendritic, virus-like, large-pore and mesoporous organosilica nanoparticles have the ability to load multiple drugs and biologics, such as peptides, proteins, siRNA and mRNA $^{2}$. Therefore, biodistribution and long-term toxicity of these new silica nanoparticle formulations will need to be tested using high-throughput assays and clinically relevant organ-on-a-chip models, in addition to clinical studies that elucidate the effects of chronic exposure to silica nanoparticles.

Proactive partnership between materials scientists, biologists, clinicians and the pharmaceutical industry will accelerate the translation of silica nanoparticles from bench to bedside.

1. Anselmo, A. C. \& Mitragotri, S. Nanoparticles in the clinic: an update post COVID-19 vaccines. Bioeng. Trans/ Med. 6, e10246 (2021).

2. Yang, Y., Zhang, M., Song, H. \& Yu, C. Silica-based nanoparticles for biomedical applications: from nanocarriers to biomodulators. Acc. Chem. Res. 53, 1545-1556 (2020).
3. Tan, A., Eskandar, N. G., Rao, S. \& Prestidge, C. A. First in man bioavailability and tolerability studies of a silica-lipid hybrid (lipoceramic) formulation: a phase I study with ibuprofen. Drug Deliv. Transl Res. 4, 212-221 (2014).

4. Bukara, K. et al. Ordered mesoporous silica to enhance the bioavailability of poorly water-soluble drugs: proof of concept in man. Eur. J. Pharm. Biopharm. 108, 220-225 (2016).

5. Meola, T. R., Abuhelwa, A. Y., Joyce, P., Clifton, P. \& Prestidge, C. A A safety, tolerability, and pharmacokinetic study of a novel simvastatin silica-lipid hybrid formulation in healthy male participants. Drug Deliv. Transl Res. 11, 1261-1272 (2021).

6. Kharlamov, A N et al. Silica-gold nanoparticles for atheroprotective management of plaques: results of the NANOM-FIM trial. Nanoscale 7, 8003-8015 (2015).

7. Kharlamov, A. N. et al. Plasmonic photothermal therapy of atherosclerosis with nanoparticles: long-term outcomes and safety in NANOM-FIM trial. Future Cardiol. 13, 345-363 (2017).

8. Rastinehad, A. R. et al. Gold nanoshell-localized photothermal ablation of prostate tumors in a clinical pilot device study. Proc. Natl Acad. Sci. USA 116, 18590-18596 (2019).

9. Zanoni, D. K. et al. Use of ultrasmall core-shell fluorescent silica nanoparticles for image-guided sentinel lymph node biopsy in head and neck melanoma. A nonrandomized clinical trial. JAMA Netw. Open 4, e211936 (2021).

10. Phillips, E. et al. Clinical translation of an ultrasmall inorganic optical-PET imaging nanoparticle probe. Sci. Trans/ Med. $\mathbf{6}$, 260ra149 (2014).

\section{Acknowledgements}

The authors are thankful for the research facilities and financial support provided by the School of Pharmacy, The University of Queensland. T.I.J. and Y.C. are thankful to The University of Queensland for the postgraduate scholarship. A.P. acknowledges NHMRC Career Development Fellowship (GNT 1146627) and strategic research funds from the School of Pharmacy. C.Y. acknowledges the Australian Research Council for support.

\section{Author contributions}

T.I.J. and Y.C. generated the first draft. T.I.J., Y.C., C.Y. and A.P. conceptualized, reviewed, and edited the manuscript.

\section{Competing interests}

The authors declare no competing interests. 